\title{
Étude du retrait en liaison avec la structure. I. Variation radiale des retraits longitudinal et tangentiel sur des placages déroulés de douglas
}

\author{
F Mothe ${ }^{1}$, B Chanson², B Thibaut², G Martin² \\ avec la collaboration technique de P Mourgues2
}

\begin{abstract}
1 INRA, station de recherches sur la qualité des bois, Champenoux 54280 Seichamps;
2 Université des sciences et techniques du Languedoc, laboratoire de mécanique générale des millieux continus, 34060 Montpellier Cedex, France
\end{abstract}

(Reçu le 21 février 1989 ; accepté le 8 septembre 1989)

\begin{abstract}
Résumé - Les retraits longitudinal et tangentiel, la densité moyenne et l'épaisseur de séries radiales de placages déroulés de douglas ont été mesurés. On observe une forte corrélation positive $(r>0,9)$ entre la densité et le retrait tangentiel. Le retrait longitudinal est lié négativement à la densité. La liaison densité-retrait est perturbée dans 2 cas particuliers :

- dans le bois le plus dense, pour lequel le retrait tangentiel est inférieur au retrait attendu; on admet que ce comportement est dû aux fissures qui apparaissent préférentiellement dans le bois dense, et limitent la déformation en s'ouvrant au séchage ;

- dans les zones de bois de compression qui présentent un retrait longitudinal anormalement élevé et un retrait tangentiel faible dans le bois final.

L'une des méthodes expérimentées pour mesurer les retraits permet d'enregistrer à tout moment au cours du séchage l'état de la déformation longitudinale et tangentielle et d'étudier ainsi la cinétique du retrait. Le retrait longitudinal commence avant et s'achève après le retrait tangentiel. On distingue 2 phases sur la courbe temps-retrait; dans la première phase $\left(T_{1}\right)_{1}$ le placage perd de l'humidite sans se déformer, le retrait ne s'effectuant qu'au cours de la seconde phase $\left(T_{2}\right)$. La longueur de la phase $T_{2}$ augmente dans les 2 directions $(L, T)$ avec la densité, alors que la phase $T 1$ se réduit lorsque la densité augmente. En définitive, la durée totale du retrait tangentiel $\left(T_{\left.1+T_{2}\right)}\right.$ est indépendante de la densité. Dans la direction longitudinale, la déformation passe en général par une phase de gonflement, mais la dimension obtenue à la fin du séchage est presque systématiquement inférieure à la dimension initiale (à l'état saturé).
\end{abstract}

retrait / structure du bois / déroulage / placage / Pseudotsuga menziesii 


\begin{abstract}
Summary - Study on shrinkage-structure relationships. l. Radial variation of longitudinal and tangential shrinkage on rotary cut Douglas veneers. Longitudinal and tangential shrinkage, average density and thickness have been measured on a 8 radial series of rotary cut veneers $(2 \times 2 \mathrm{~cm})$ using the experimental procedure described in figure 1 . The results are presented on radial profiles (fig 3). There was a strong positive correlation ( $r>0.9)$ between tangential shrinkage and density. The phenomenogical relationship between longitudinal shrinkage and density was negative and lower than the one mentioned above (fig 4). Two cases deviate from this density/shrinkage relationship:

- zones characterised by high density present lower than expected tangential shrinkage. It is assumed that this phenomenon is due to cracks which mostly appear in. late-wood and reduce the strain by opening during drying;

- compression wood (marked BC in fig 3) shows abnormally high longitudinal and low tangential shrinkage in late-wood.

The method of shrinkage measurement described in figure 2 can give the tangential and longitudinal deformations at any time during drying, thus allowing the study of shrinkage kinetics. Table $\mathrm{N}$ shows the mean values of the kinetic parameters as defined in figure 5. Longitudinal shrinkage is induced first, and then, tangential shrinkage acts simultaneously with the longitudinal one. The longitudinal shrinkage subsequently completes the deformation. Two phases were distinguished on the shrinkage vs time curve: during the first $\left(T_{1}\right)$, the veneer loses humidity without warping, then shrinkage occurs during the second phase $\left(T_{2}\right)$. As the density increases, duration of the $T_{2}$-phase increases for both directions $(L, T)$ whereas the duration of the $T_{1}$ phase is reduced. Finally, the total duration of tangential shrinkage (too $\left.=T_{1}+T_{2}\right)$ is not dependent on the density (table $\mathrm{N}$ ). In the longitudinal direction, the strain usually exhibits a swelling phase but the final dimensions of a seasoned specimen are nearly always lower than a green specimen.
\end{abstract}

\title{
shrinkage / wood structure / peeling process / veneer / Pseudotsuga menziesii
}

\section{INTRODUCTION}

Les déformations des pièces de bois sous l'effet des variations hygrométriques peuvent entrainer une dévalorisation importante du produit. Les conséquences en sont d'autant plus néfastes que l'hétérogénéité du matériau est importante; les variations dimensionnelles s'accompagnent alors de déformations par gauchissement ou vrillage qui conduisent éventuellement à l'élimination des pièces défectueuses.

Les placages obtenus par tranchage ou déroulage et les panneaux à base de placages sont particulièrement sensibles à ces défauts: une faible différence de retrait entre les 2 faces d'un contreplaqué suffit à provoquer un tuilage du panneau au séchage qu'il sera difficile de corriger et qui risquera de réapparaître, sous l'ef- fet de variations hygrométriques après la mise en place.

Pour prévenir de tels défauts, il serait utile de prédire la déformation d'une pièce de bois (massif ou reconstitué) à partir d'une description suffisamment précise de la structure et de l'environnement. Pour cela, il est nécessaire de connaître, en tout point du matériau et à tout instant, l'humidité du bois, ses caractéristiques mécaniques et son état de contraintes. La détermination des lois de variation des retraits (en l'absence de contraintes) avec la structure, notamment avec la position dans le cerne annuel, est une étape nécessaire pour y parvenir.

Dans l'étude des liaisons retraitsstructure, les expérimentateurs se heurtent à la difficulté d'usiner des éprouvettes de dimension suffisamment 
importante pour ne pas nuire à la précision de la mesure, et suffisamment réduite pour garantir l'homogénéité de la structure dans l'éprouvette. Le compromis généralement adopté consiste à travailler sur des segments de carottes de sondage dont la dimension radiale excède la largeur des cernes (Polge, 1964; Polge et al, 1973) ou sur des éprouvettes usinées spécifiquement en petite quantité (Harris et Meylan, 1965; Pentoney, 1953). L'existence d'une liaison plus ou moins étroite entre densité du bois et retraits transverses (tangen-

Tableau I. Retraits totaux (saturation $\rightarrow$ anhydre) observés sur le douglas par differents auteurs.

\begin{tabular}{|c|c|c|c|c|c|c|}
\hline (1) & Densité & tangentiel & $\begin{array}{c}\text { Retrait \% } \\
\text { radial }\end{array}$ & axial & Auteurs & \\
\hline $\begin{array}{c}\text { BI } \\
\text { BF } \\
\text { moy. }\end{array}$ & $\begin{array}{l}0,3 \\
1.1 \\
0,54\end{array}$ & $\begin{array}{r}6,6 \\
14,4 \\
8,9\end{array}$ & $\begin{array}{r}0.6 \\
14.2 \\
4.7\end{array}$ & : & QUIRK 75 & \multirow{3}{*}{$\begin{array}{l}\text { cités } \\
\text { par } \\
\text { QUIRK } \\
82\end{array}$} \\
\hline $\begin{array}{c}B I \\
B F \\
\text { moy. }\end{array}$ & $\begin{array}{l}0,4 \\
0,3 \\
-\end{array}$ & $\begin{array}{l}4,8 \\
7,2 \\
7,2\end{array}$ & $\begin{array}{l}2,4 \\
8,9 \\
4,4\end{array}$ & : & PENTONEY 53 & \\
\hline $\begin{array}{l}B I \\
B F \\
\text { moy. }\end{array}$ & $\begin{array}{l}0,3 \\
0,8 \\
:\end{array}$ & $\begin{array}{r}5,7 \\
10,9 \\
8,2\end{array}$ & $\begin{array}{l}2,9 \\
9,9 \\
5,2\end{array}$ & $\dot{-}$ & VINTILA 39 & \\
\hline $\begin{array}{l}B I \\
B F\end{array}$ & $\begin{array}{l}0,28 \\
0.69\end{array}$ & $\begin{array}{l}5,5 \\
8,5\end{array}$ & - & $\dot{-}$ & \multicolumn{2}{|c|}{$\begin{array}{l}\text { divers cités par } \\
\text { LUTZ } 74\end{array}$} \\
\hline $\begin{array}{l}B C \\
B N\end{array}$ & : & $\begin{array}{l}4,2 \\
5,9\end{array}$ & $\begin{array}{l}2,5 \\
3,4\end{array}$ & $\begin{array}{l}0,67 \\
0,17\end{array}$ & $\begin{array}{l}\text { PILLOW et } \\
\text { LUXFORD } 37\end{array}$ & $\begin{array}{l}\text { citès } \\
\text { par }\end{array}$ \\
\hline $\begin{array}{l}\mathrm{BC} \\
\mathrm{BN}\end{array}$ & $\dot{-}$ & - & : & $\begin{array}{l}0,2-1,1 \\
0,1-0,2\end{array}$ & $\begin{array}{l}\text { TRENOELENBURG } \\
32\end{array}$ & $\begin{array}{l}\text { TIMELL } \\
86\end{array}$ \\
\hline $\begin{array}{l}B J \\
B A\end{array}$ & $\begin{array}{l}0,34-0,43 \\
0,44-0,49\end{array}$ & $\begin{array}{l}8,5 \\
9.8\end{array}$ & $\begin{array}{l}4,9 \\
6,5\end{array}$ & $\begin{array}{l}0,33-0,49 \\
0,38-0,48\end{array}$ & \multicolumn{2}{|l|}{$\begin{array}{c}\text { POLGE } \\
64\end{array}$} \\
\hline $\begin{array}{l}\text { AT } \\
\text { AE }\end{array}$ & $\begin{array}{l}0,37-0,37 \\
0,37-0,42\end{array}$ & $\begin{array}{l}5,2-6,2 \\
4,9-6,3\end{array}$ & $\begin{array}{l}2,8-3,1 \\
2,6-3,5\end{array}$ & $\begin{array}{l}0,35 \cdot 0,26 \\
0,17 \cdot 0,01\end{array}$ & \multicolumn{2}{|c|}{$\begin{array}{c}\text { POLGE et al. } \\
73\end{array}$} \\
\hline & 0,39 & $\begin{array}{l}6,9 \\
7,8\end{array}$ & $\begin{array}{l}4,0 \\
5,0\end{array}$ & - & $\begin{array}{l}\text { POLGE } \\
\text { divers }\end{array}$ & $\begin{array}{l}\text { cités par } \\
\text { POLGE } 63\end{array}$ \\
\hline & $\dot{-}$ & $\begin{array}{l}7,4 \\
8,0 \\
4,9 \\
8,7\end{array}$ & $\begin{array}{l}4,4 \\
4,7 \\
3,7 \\
5,7\end{array}$ & 0,5 & $\begin{array}{l}\text { moyenne USA } \\
\text { KNIGGE S8 } \\
\text { OE ALMEIDA } 55 \\
\text { HORVAT } 53\end{array}$ & $\begin{array}{l}\text { cités } \\
\text { par }\end{array}$ \\
\hline & $\dot{-}$ & $\begin{array}{l}8,8 \\
7,2\end{array}$ & $\begin{array}{l}4,8 \\
4,1\end{array}$ & $\begin{array}{l}0,6 \\
0,6\end{array}$ & GIORDANO & $\begin{array}{l}\text { GIORDANO } \\
59\end{array}$ \\
\hline & $0,45-0,61 \mid$ & $4,2-9,0$ & $2,7-5,6$ & $\cdot$ & \multicolumn{2}{|c|}{ divers par TAKAHASHI 83} \\
\hline
\end{tabular}

(1) $\mathrm{Bl} / \mathrm{BF}$ : bois initial / bois tinal; $\mathrm{BC} / \mathrm{BN}$ : bois de compression / normal; $\mathrm{BJ} / \mathrm{BA}$ : bois juvénile / bois adulte; $A T$ / $A E$ : arbres témoins / arbres élagués. 
tiel ou radial) a ainsi été mise en évidence par ces auteurs. La prise en compte de données anatomiques simples, telles que la texture du cerne ou le nombre de rayons ligneux en complément de la densité, permet en général d'améliorer la prédiction du retrait (Aubert, 1987). En ce qui concerne le douglas, les compilations des travaux antérieurs (Quirk, 1982; Lutz, 1974, tableau I) montrent que les retraits transverses sont toujours plus importants dans le bois final que dans le bois initial.

Les conséquences de la liaison retrait tangentiel - densité sur la planéité de placages hétérogènes ont été étudiées par Luxeuil (1983) qui observe une liaison entre l'hétérogénéité du cerne et le rayon de courbure du placage sec. Les ondulations d'un placage après séchage sont imputables, en grande partie, aux variations locales du retrait longitudinal, mais un placage dont le retrait global est élevé n'est pas forcément ondulé. Marchal (1983) observe même une liaison négative entre retrait tangentiel mesuré sur carotte et défauts de planéité de placages tranchés de chêne.

À l'inverse des retraits transverses, le retrait longitudinal s'avère plus élevé dans le bois tendre que dans le bois dense (FPL, 1960; Kollmann et Cote, 1968; Timell, 1986). Hann, observe (1969) des coefficients de corrélation élevés mais de signes variables entre retrait longitudinal et densité des éprouvettes issues du même arbre. La plupart des auteurs admettent que la densité n'est pas suffisante à elle seule pour expliquer la variabilité du retrait longitudinal.

II est incontestable, cependant, que celui-ci est affecté par la microstructure des parois cellulaires. Selon la théorie de Barber et Meylan, (1964); Harris et Meylan, (1965), la paroi cellulaire peut être assimilée d'un point de vue hygroscopique à une matrice amorphe isotrope renforcée par une armature constituée de microfibrilles de cellulose cristallisée. En présence d'eau, le gonflement de la matrice serait uniforme s'il n'était pas contrarié par les microfibrilles dont la longueur est supposée invariable. L'orientation quasi longitudinale des microfibrilles dans la couche $S_{2}$ de la paroi cellulaire (la plus importante par son épaisseur) explique ainsi l'anisotropie entre les retraits transverses et le retrait longitudinal (tableau I).

De la même façon, les variations locales du retrait longitudinal (ou des retraits transverses) peuvent être attribuées à des différences d'inclinaison moyenne des microfibrilles de la couche $S_{2}$. A l'intérieur d'un cerne, l'angle des microfibrilles ( $A M F$ ) décroît rapidement dans les premières trachéides du bois initial (Fujisaki et Shibuya, 1986; Siripatanadilok et Leney, 1985); on observe souvent un écart de plus de $10^{\circ}$ entre l'AMF du bois initial et celui du bois final (Boyd, 1974; Park, 1984). Le fort retrait longitudinal du bois de compression (tableau I) s'explique également par un AMF anormalement élevé. Le tableau II résume les résultats obtenus sur du douglas par El Osta et Wellwood (1972).

À l'examen de la littérature, on constate que 2 approches très différentes ont été utilisées pour étudier les

Tableau II: Angles des microfibrilles mesurés sur du bois de douglas par El Osta et Wellwood (1972).

\begin{tabular}{llc}
\hline $\begin{array}{c}\text { Angle } \\
\text { des microfibrilles }\end{array}$ & $\begin{array}{l}\text { Bois } \\
\text { initial }\end{array}$ & $\begin{array}{c}\text { Bois } \\
\text { final }\end{array}$ \\
\hline Bois normal & $21,6^{\circ}$ & $12,9^{\circ}$ \\
Bois de compression & $28,2^{\circ}$ & $17,4^{\circ}$ \\
\hline
\end{tabular}


liaisons retraits-structure : l'approche "fondamentale», qui débouche sur des théories expliquant de façon extrêmement fine le mécanisme des retraits, et l'approche "statistique" qui vise à définir des lois de variation des retraits avec des paramètres descriptifs simples de la structure du matériau (densité du bois, largeur des cernes, etc). Pour parvenir à modéliser le comportement au séchage d'une pièce de bois en fonction de sa structure, les 2 approches peuvent être adoptées mais aucune n'est parfaitement satisfaisante: il n'est pas envisageable de mesurer en routine l'AMF et les lois statistiques obtenues sur éprouvettes normalisées ou sur carottes de sondage ne permettent d'expliquer qu'une faible part de la variabilité des retraits. Nous proposons ici une démarche expérimentale intermédiaire entre ces 2 approches: la confection par déroulage d'éprouvettes de structure homogène devrait permettre d'affiner les lois statistiques classiques et, ultérieurement, de valider les théories en vigueur grâce notamment à la prise en compte de l'aspect cinétique des retraits.

\section{MATÉRIEL ET MÉTHODES}

Le déroulage en faible épaisseur permet d'obtenir facilement des éprouvettes adaptées à l'étude de la variabilité intracerne des retraits longitudinal et tangentiel: dimension radiale faible devant la largeur de cerne mais section longitudinale-tangentielle importante. Le retrait radial est, en revanche, difficilement mesurable sur des placages et nous nous abstiendrons d'y faire référence dans cette premiere partie. Pour les utilisateurs de placages, que le retrait radial intéresse relativement peu, ce procédé s'avère particulièrement adapté, étant donné que la forme et le procédé d'obtention des éprouvettes sont comparables à ceux du produit réel.

\section{Matériel végétal :}

Trois disques épais de $2 \mathrm{~cm}$ et exempts de nœuds ont été prélevés sur 3 douglas issus d'une plantation experimentale de 20 ans appartenant à la station d'amélioration des arbres forestiers de I'INRA (plantation de Peyrat-le-Château, Limousin).

\section{Déroulage :}

Les éprouvettes ont été déroulées - après plusieurs mois de stockage en immersion au laboratoire de mécanique générale des milieux continus (université de Montpellier) sur une machine expérimentale d'étude du déroulage (Thibaut, 1988). Le déroulage a été effectué à vitesse angulaire constante (2 tours/s), sans barre de pression, et pour des épaisseurs de 0,4 et $0,6 \mathrm{~mm}$.

Le ruban de placage déroule a été découpé de façon à reconstituer des séries radiales d'une centaine d'éprouvettes (placages de $2 \mathrm{~cm} \times 2 \mathrm{~cm}$ ) selon la procédure décrite figure 1 .

\section{Mesure des retraits sur placages :}

La mesure des retraits longitudinal et/ou tangentiel a ensuite été effectuée sur chacun des placages obtenus. Suivant l'arbre considéré, 3 méthodes ont été expérimentées (tableau III) :

\section{Méthode manuelle ( 3 séries radiales - 0,4 mm)}

II s'agit simplement de mesurer au palmer la longueur de chaque placage avant et après séchage à l'air libre. Cette méthode rudimentaire, probablement entachee d'un "effet manipulateur" non négligeable a finalement donné des résultats cohérents avec les autres mesures. Nous ne l'avons utilisée que pour la mesure du retrait tangentiel.

\section{Méthode optique (4 séries radiales - $0,6 \mathrm{~mm}$ )}

Le placage saturé d'eau est déposé au contact d'une equerre métallique solidaire de la platine d'un microscope à réflection. L'arête du placage, grossie environ 300 fois, 


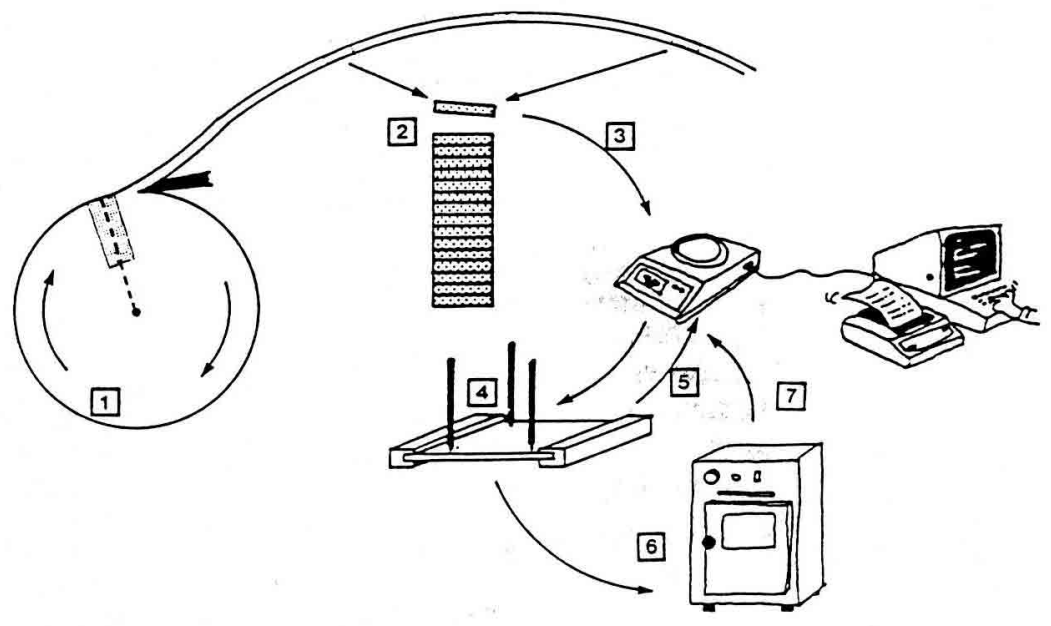

Fig 1. Procédure expérimentale. 1: déroulage; 2: reconstitution des séries radiales; 3 : pesée à l'état saturé; 4: mesure des retraits; 5: pesée à la fin du séchage; 6: déshydratation; 7: pesée à l'état anhydre.

est visualisée sur un écran gradué; on mesure alors avec une précision théorique de quelques $10^{-2} \mathrm{~mm}$ le déplacement de la platine nécessaire pour faire apparaître au même emplacement sur l'écran l'arête d'une cale métallique substituée au placage. La mesure est répétée après séchage à l'air ambiant et la différence entre les 2 valeurs rapportée à la dimension humide est égale au retrait. Cette $m$ s'est avérée satisfaisante pour la détermination du retrait tangentiel, mais insuffisamment précise pour la mesure des variations de faible amplitude du retrait longitudinal.

\section{Méthode numérique (2 séries ra- diales $-0,6 \mathrm{~mm}$}

La m numérique est beaucouup plus lente que les précédentes car chaque placage doit être séché individuellement. Elle est aussi beaucoup plus précise et offre davantage d'informations, en particulier sur la cinétique des retraits. Le montage et la mise au point de cette expérience ont été réalisés au LMGMC par G Martin.
Le placage humide est fixé sur un dispositif constitué de 2 pointes mobiles distantes de $15 \mathrm{~mm}$ d'une $3^{\theta}$ pointe fixe, laquelle marque le sommet d'un triangle isocèle rectangle (fig 2). L'ensemble est placé dans une enceinte thermorégulée à $45^{\circ} \mathrm{C} \pm 0,1^{\circ} \mathrm{C}$ par chauffage infrarouge et ventilation forcée. L'hygrométrie peut être supposée constante étant donné le volume de l'enceinte $(0,35$ $\mathrm{m}^{3}$ ) et l'existence d'ouvertures autorisant le renouvellement d'air. Pendant le séchage, la distance de la pointe fixe a chacune des 2 pointes mobiles évolue avec les retraits tangentiel et longitudinal du placage. Cette distance est transmise périodiquement à un micro-ordinateur par l'intermédiaire de capteurs extensométriques. Chaque seconde, 1000 données sont ainsi saisies sur les 2 voies et moyennées de façon à filtrer le bruit de fond. La précision de la mesure est de l'ordre de $0,1 \mu \mathrm{m}$. On obtient ainsi 2 courbes temps-déformation (longitudinale et tangentielle) qui présentent 2 paliers dont la différence représente le retrait du placage sur $15 \mathrm{~mm}$ entre l'état saturé et le point d'équilibre hygroscopique du bois dans l'enceinte. 


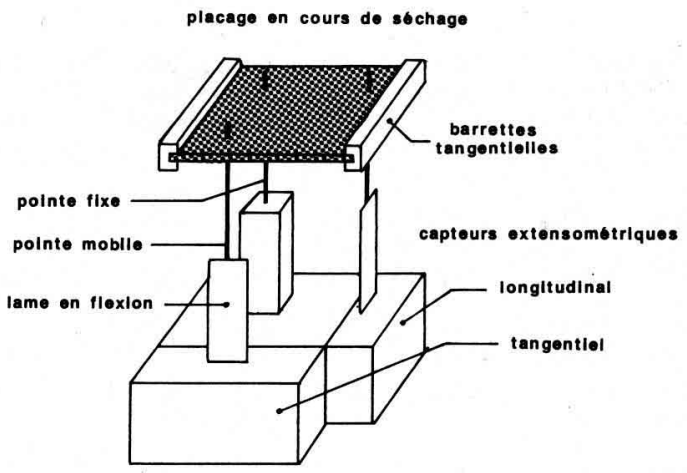

Fig 2. Représentation schématique du dispositif utilisé pour la mesure des retraits longitudinal et tangentiel (méthode numérique).

\section{Mesures après séchage}

L'humidité des placages en fin de séchage a été contrôlée par pesée de quelques éprouvettes à la sortie de l'enceinte et après déshydratation complète. Elle s'élève à $6,2 \%$ dans la dernière $m$ (c) contre environ $9,5 \%$ dans les 2 premières.

Après stabilisation à l'état sec à l'air (environ $9.5 \%$ d'humidité) les données complémentaires suivantes ont été relevées pour chaque placage :

- épaisseur, mesurée au palmer,

- poids,

- densité déterminée par le rapport (poids/ volume).

Note: quelle que soit la $\mathrm{m}$ de mesure, les retraits sont exprimés en proportion de la dimension à l'état saturé :

$$
R_{h}=\frac{D_{S}-D_{h}}{D_{S}} \times 100
$$

où $D_{s}$ est la dimension à l'état saturé et $D_{h}$ la dimension à l'humidité finale $H(H=9,5$ ou $6,2 \%$ suivant la $m$ ). Quelle que soit $H$, la densité est mesurée à $9,5 \%$ d'humidité.

\section{RÉSULTATS ET DISCUSSIONS}

\section{Valeurs moyennes obtenues}

Le tableau III présente les caractéristiques moyennes des disques déroulés et le nombre de séries radiales analysées dans chaque cas. Le retrait longitudinal ne dépasse jamais $8 \%$; la moyenne sur une série est inférieure à $3 \%$. Suivant la $\mathrm{m}$ de mesure et l'echantillon concernés, le retrait tangentiel moyen oscille entre 4,86 et $7,10 \%$. En admettant que le retrait évolue linéairement avec I'humidité en dessous du point de saturation des fibres $(28 \%$ pour le douglas selon le cahier du CTBA, 1986), le retrait tangentiel total (dimension du placage à saturation - dimension du placage anhydre) varierait entre 6,24 et $10,75 \%$ (tableau III).

\section{Profils radiaux}

Chaque série radiale est constituée d'une centaine de placages adjacents. L'ensemble des mesures effectuées sur une série radiale est représenté dans la figure 3 sous forme de profils.

\section{Liaison retraits-densité}

Dans la plupart des cas, il existe une forte liaison entre retrait tangentiel et densité moyenne du placage (fig 3 et 4 ). 
Tableau III. Résultats moyens obtenus dans les expériences de séchage.

\begin{tabular}{|c|c|c|c|c|c|c|c|c|c|c|c|}
\hline \multirow{2}{*}{\multicolumn{2}{|c|}{$\begin{array}{c}\text { Eprouvette et } \\
\text { secteur radial (1) }\end{array}$}} & \multicolumn{2}{|c|}{ Retrait (\%) } & \multirow{2}{*}{$\begin{array}{c}\text { Densité } \\
\text { sec à } \\
\text { l'air }\end{array}$} & \multirow{2}{*}{$\begin{array}{c}r \\
(R, D) \\
(4)\end{array}$} & \multirow{2}{*}{$\begin{array}{l}\text { Lar- } \\
\text { geur } \\
\text { cernes } \\
(\mathrm{mm})\end{array}$} & \multirow{2}{*}{$\begin{array}{c}\mathrm{Nb} \\
\text { pla- } \\
\text { cages }\end{array}$} & \multirow{2}{*}{$\begin{array}{l}\text { Mé- } \\
\text { thode } \\
\text { (5) }\end{array}$} & \multirow{2}{*}{$\begin{array}{c}\text { Tempéra- } \\
\text { ture } \\
\text { séchage } \\
\left({ }^{\circ} \mathrm{C}\right)\end{array}$} & \multirow{2}{*}{$\begin{array}{c}\text { Humidi- } \\
\text { té } \\
\text { finale } \\
(\%)\end{array}$} & \multirow{2}{*}{$\begin{array}{c}\text { Épais- } \\
\text { seur } \\
(\mathrm{mm})\end{array}$} \\
\hline & & $\begin{array}{l}\text { Total } \\
\text { (2) }\end{array}$ & $\begin{array}{l}\text { observé } \\
\text { (3) }\end{array}$ & & & & & & & & \\
\hline \multirow{7}{*}{$\begin{array}{l}\text { Retrait } \\
\text { tangentiel }\end{array}$} & 034 A3 1 & 8,55 & $5,65 \pm 2,0$ & $0,49 \pm 0,20$ & 0,97 & 7,1 & 82 & \multirow{3}{*}{$P$} & \multirow{3}{*}{20} & \multirow{3}{*}{$9,5 \%$} & \multirow{3}{*}{0,4} \\
\hline & 3 & 9,05 & $5,98 \pm 1,8$ & $0,48 \pm 0,20$ & 0,98 & 4,0 & 82 & & & & \\
\hline & 4 & 9,07 & $5,99 \pm 1,5$ & $0,48 \pm 0,15$ & 0,96 & 4,3 & 83 & & & & \\
\hline & 718 C3 1 & 9,26 & $6,12 \pm 1,8$ & $0,52+0,16$ & 0,65 & 6,0 & 101 & \multirow{4}{*}{0} & \multirow{4}{*}{90} & \multirow{4}{*}{$9,5 \%$} & \multirow{4}{*}{0,6} \\
\hline & 2 & 10,03 & $6,63 \pm 2,0$ & $0,51 \pm 0,17$ & 0,63 & 6,0 & 101 & & & & \\
\hline & 3 & 10,75 & $7,10 \pm 2,1$ & $0,53 \pm 0,22$ & 0,91 & 4,6 & 102 & & & & \\
\hline & 4 & 10,43 & $6,89 \pm 1,9$ & $0,51 \pm 0,19$ & 0,93 & 5,0 & 102 & & & & \\
\hline \multirow{3}{*}{$\begin{array}{l}\text { Retrait } \\
\text { axial }\end{array}$} & $092 \mathrm{C} 34$ & 6,24 & $4,86 \pm 1,5$ & $0,45 \pm 0,17$ & 0,85 & 5,5 & 110 & $N$ & 45 & $6,2 \%$ & 0,6 \\
\hline & 718 C3 1 & - & $0,25 \pm 0,2$ & $0,52 \pm 0,16$ & $-0,18$ & 6,0 & 100 & $N$ & 40 & $6,2 \%$ & 0,6 \\
\hline & $092 \mathrm{C3} 4$ & - & $0,14 \pm 0,1$ & $0,45 \pm 0,17$ & $-0,65$ & 5,5 & 110 & $\mathrm{~N}$ & 45 & $6,2 \%$ & 0,6 \\
\hline
\end{tabular}

(1) $n^{\circ}$ arbre: 034, 092, 718; $n^{\circ}$ disque: A3, C3; $n^{\circ}$ secteur radial: 1, 2, 3, 4 (2) estimation du retrait de l'état saturé à l'état anhydre; (3) retrait réel entre l'état saturé et l'humidité finale observée; (4) coefficient de corrélation entre retrait mesuré et densité; (5) méthode de mesure: $\mathbf{P}=$ palmer, $0=$ optique, $N=$ numérique.

Le coefficient de corrélation est souvent $>0,9$ (tableau III). Pour les densités comprises entre 0,2 et 0,7 on decrit assez bien la loi de variation du retrait tangentiel entre l'état saturé et I'humidité finale à la sortie du séchoir $(6,2$ ou $9,5 \%)$ par une relation simple du type:

Retrait tangentiel $(\%)=(10 \times$ densité) $+K$

où $K$ varie entre 2,5 et $4 \%$ suivant la $m$ de mesure et/ou les conditions de séchage.

Le retrait longitudinal est, au contraire, lié négativement à la densité. La relation retrait longitudinal - densité ne parait pas être aussi simple que celle qui lie la densité au retrait tangentiel. Les données fournies par Polge (1964) vont dans le même sens: le retrait longitudinal, mesuré entre l'état vert et l'état sec à l'air, sur des carottes de douglas, varie inversement avec la densité du cerne, mais Polge montre que les éprouvettes de bois dense rattrapent leur retard lorsque le séchage est poursuivi jusqu'à déshydratation complète; le retrait longitudinal total s'avère, en définitive, indépendant de la densité.

A l'examen des figures 3 et 4 , on remarquera que le retrait tangentiel s'écarte de la droite de régression dans 2 cas:

- Iorsque la densité est trop forte (supérieure à 0,7 dans la figure 4), le retrait tangentiel se stabilise ou décroît même dans certaines expériences. Ceci pourrait s'expliquer par la présence de fissures dans le bois le plus 

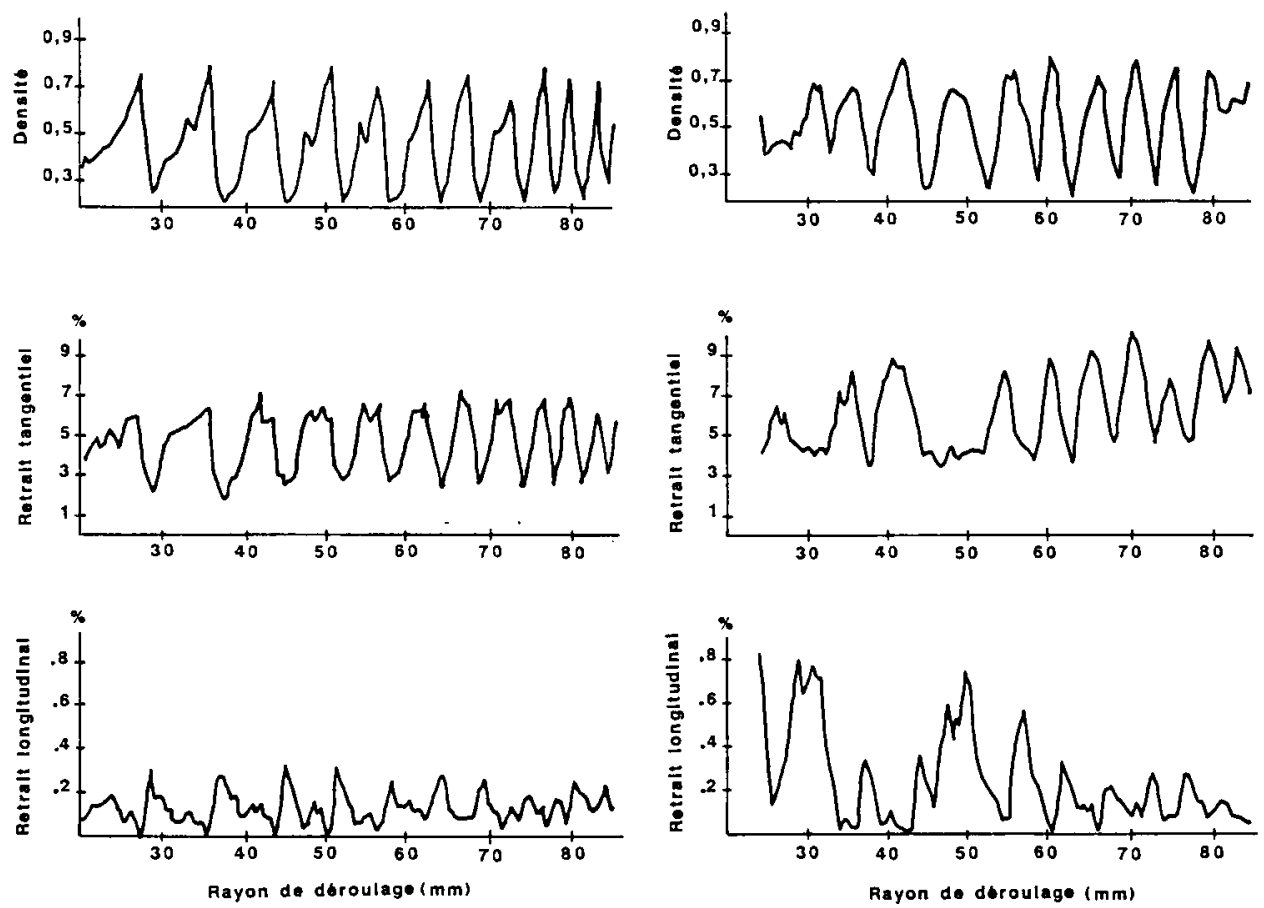

Fig 3. Profils radiaux de la densité et des retraits tangentiel et longitudinal obtenus pour deux éprouvettes: $092 \mathrm{C} 3$, secteur 4 à gauche $-718 \mathrm{C} 3$, secteur 1 à droite.
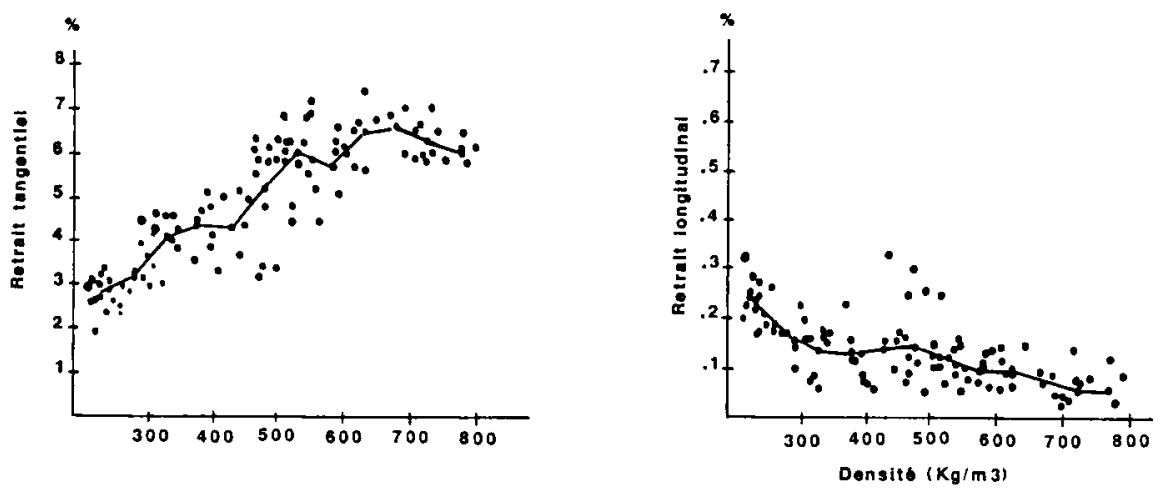

Fig 4. Évolution des retraits tangentiel et longitudinal avec la densité pour l'éprouvette 092C3, secteur 4.

dense. On sait en effet que, dans le processus de déroulage, il existe un risque de fissuration de la face inférieure (face ouverte) du placage; les fissures n'apparaissent que rarement lorsque l'épaisseur de coupe est faible comme c'est le cas ici; cependant le risque s'accroît lorsque la densité s'élève (Thibaut, 1988; Mothe, 1988). Dans le cas présent, un examen super- 
ficiel a mis en évidence l'existence de fissures dans les placages les plus denses de l'éprouvette 092C3 dont les retraits sont représentés sur la figure 4 . Les travaux de Marchal (1989) devraient permettre de vérifier l'influence du taux de fissuration sur le retrait mais il paraît raisonnable d'admettre que la mesure du retrait tangentiel est sousestimée lorsque le placage présente des fissures susceptibles de s'ouvrir au séchage, ce qui correspondrait aux résultats expérimentaux.

- dans certains cernes de l'éprouvette $718 \mathrm{C} 3$ (fig 3 ), le retrait tangentiel reste constamment à un niveau bas, correspondant au retrait habituel du bois de printemps, alors que le retrait longitudinal s'accroît très fortement. L'examen en microscopie optique des cernes correspondants a permis de mettre en évidence dans ces zones du bois de compression, caractérisé par de larges méats intercellulaires. La présence de bois de compression suffit à expliquer les anomalies du retrait longitudinal, comme en témoignent les résultats obtenus sur du douglas par Trendelenburg (1932) et Pillow et Luxford (1937), cités par Timell (1986). Ces résultats ne faisaient cependant pas apparaître de différences sensibles pour les retraits transverses entre bois normal et bois de compression.

Contrairement à ce que l'on aurait pu attendre, le bois le plus proche de la moelle ne présente pas de particularités du point de vue des retraits, dès lors qu'il est exempt de bois de compression. La comparaison entre les retraits du bois juvénile et du bois adulte de douglas établie par Polge (1964) confirme ce résultat (tableau 1). Notons cependant que les tout premiers cernes ( 2 à 3 cernes) font partie du noyau de déroulage et echappent de ce fait à l'analyse.

\section{Cinétique du séchage}

L'état final d'une pièce de bois soumise à un changement d'humidité dépend évidemment des retraits mais également de la vitesse relative des déformations dans chaque direction et en chaque point de la pièce. Bien que la $m$ (c) décrite ci-dessus ne permette pas de connaître les variations d'humidité au cours du séchage, elle offre des informations suffisamment détaillées pour étudier et comparer la cinétique des retraits longitudinal et tangentiel. Les résultats présentés ici ne concernent que l'éprouvette $092 \mathrm{C} 3$ sur laquelle les déformations ont été mesurées simultanément dans les 2 directions tout au long du séchage.

L'allure générale d'une courbe tempsretrait évoque une fonction arc-tangente: la vitesse de déformation s'accroît très lentement dans la première phase, au cours de laquelle s'effectue le «ressuyage", puis s'accélère rapidement durant la phase de retrait proprement dite, avant de décroître à nouveau, jusqu'à l'obtention d'une palier (fig 5). Pour paramètrer une telle courbe, nous avons choisi d'utiliser les variables suivantes: $R_{\infty}$ : retrait maximal observé; $R_{1 / 2}=R_{\infty / 2}$ : demi-retrait; $T_{1}$ : durée de la phase de «ressuyage»; $T_{2}$ : durée de la phase de retrait; $t_{\infty}=T_{1}+T_{2}$ : temps de séchage jusqu'au retrait; $t_{1 / 2}=T_{1}+T_{2 / 2}$ : temps de séchage jusqu'au demi-retrait, en admettant la symétrie de la courbe dans la phase de retrait; $a=t_{\infty} / t_{1 / 2}$ : coefficient de «retard». Lorsque a vaut 0,5 , le retrait s'amorce instantanément et $T_{1}=0$. Le coefficient a tend vers 0 lorsque $T_{1}$ augmente.

D'un point de vue pratique, les paramètres $T_{1}$ et $T_{2}$ sont estimés au moyen de la courbe temps-retrait en admettant que la phase de retrait s'amorce à $5 \%$ et s'achève à $95 \%$ de la déformation totale (fig 5 ). 


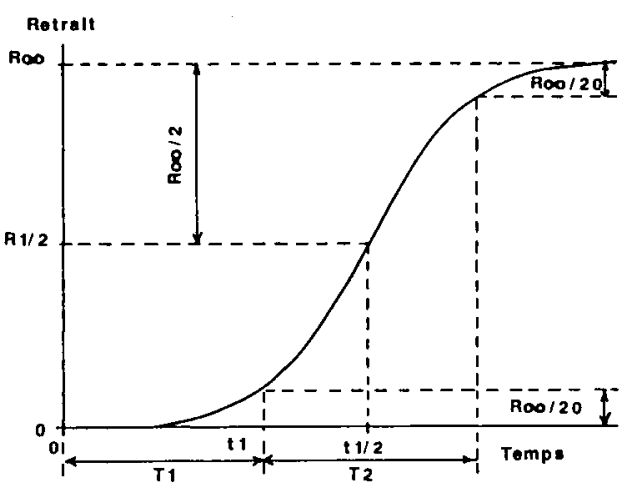

Flg 5. Définition des symboles utilisés pour paramétrer les courbes temps - retrait.

Le tableau IV montre, pour l'éprouvette $092 \mathrm{c} 3$, les valeurs moyennes de ces parametres dans les 2 directions ainsi que leurs coefficients de corrélation avec la densité. On peut en tirer les conclusions suivantes:

Tableau IV. Caractéristiques cinétiques du retrait dans les directions longitudinale et tangentielle. Valeurs moyennes sur l'éprouvette 092C3 des paramètres décrits par la figure 5 et coefficients de corrélation avec la densité (82 données).

\begin{tabular}{lccc}
\hline & \multicolumn{2}{c}{ Direction } \\
& & $\begin{array}{c}\text { congitu- } \\
\text { dinale }\end{array}$ & $\begin{array}{c}\text { tangen- } \\
\text { tielle }\end{array}$ \\
\hline Durée de "ressuyage" & $T_{1}$ & $5^{\prime}$ & $8^{\prime}$ \\
& $(-0,37)$ & $(-0,64)$ \\
Durée du retrait & $T_{2}$ & $15^{\prime}$ & $9^{\prime}$ \\
& $(+0,65)$ & $(+0,72)$ \\
Temps total de séchage & too & $20^{\prime}$ & $17^{\prime}$ \\
& $(+0,60)$ & $(+0,22)$ \\
Période de demi-retrait & $t 1 / 2$ & $12^{\prime}$ & $12^{\prime}$ \\
& $(+0,39)$ & $(-0,19)$ \\
Coefficient de "retard" & a & 0,63 & 0,73 \\
& & $(-0,51)$ & $(-0,86)$ \\
Retrait observé & $r \%$ & $0,14 \%$ & $4,8 \%$ \\
& & $(-0,72)$ & $(+0,84)$ \\
\hline
\end{tabular}

(1) coefficient de correlation avec la densité du bois. Tous les coefficients sont significatifs au seuil de $1 \%$ a l'exception de $t_{\infty}$ et $t_{1 / 2}$ la direction tangentielle
- le retrait longitudinal s'amorce avant le retrait tangentiel et se poursuit plus longtemps;

- après douze minutes de séchage, la moitié de la déformation est atteinte dans les 2 directions;

- la phase de "ressuyage» $\left(T_{1}\right)$ est d'autant plus réduite et la phase de retrait $\left(T_{2}\right)$ d'autant plus longue que le placage est dense; cela s'explique par la plus grande quantité d'eau libre susceptible d'être contenue dans les trachéides larges du bois de printemps; la phase de retrait ne commence que lorsque l'eau libre est évacuée, les échanges s'effectuant alors en phase gazeuse et étant ralentis dans les tracheides les plus étroites;

- la durée totale du retrait $\left(t_{\infty}\right)$ est indépendante de la densité du placage dans le sens tangentiel alors qu'elle s'accroît sensiblement pour la direction longitudinale, notamment dans les placages traversant une limite de cerne.

Alors que le retrait tangentiel évolue de façon monotone avec un point d'inflexion aux alentours de $R_{1 / 2}$, la déformation longitudinale change de signe au cours du séchage, en particulier dans les placages contenant du bois dense: on observe une premiere phase de retrait suivie d'un gonflement, parfois suffisant pour que la dimension de l'éprouvette en cours de séchage dépasse provisoirement la dimension initiale, puis une nouvelle phase de retrait (fig 6).

A de rares exceptions près, le résultat est positif (retrait), mais il est probable que si le séchage avait été interrompu peu de temps avant la mise à l'équilibre (à l'humidité de 12\% par exemple), beaucoup d'éprouvettes constituées de bois final auraient manifesté un retrait longitudinal négatif. C'est ainsi que Koehler (1931) cite par Kollmann et Cote (1968) puis Barber et Meylan (1964) observaient un retrait sur du douglas, négatif entre 

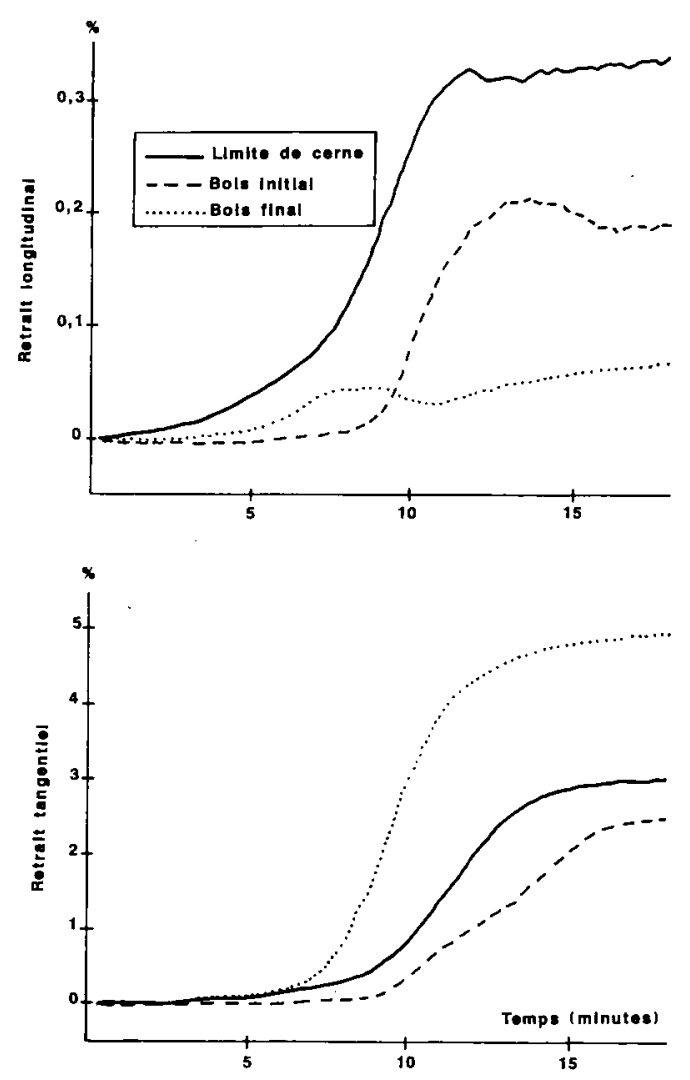

Fig 6. Trois exemples de courbes temps - retrait obtenues pour deux placages homogènes composés de bois initial (BI) et de bois final (BF) et pour un placage hétérogène traversé par une limite de cerne (LC).

l'état vert et l'état sec à l'air, puis positif lorsque le séchage était poussé jusqu'à l'état anhydre.

La théorie de la matrice amorphe de Barber et Meylan offre une explication de ce phénomène: lorsque le bois est saturé d'eau, la matrice de cellulose amorphe est peu rigide; sa rigidité s'accroît au fur et à mesure que l'eau est évacuée. En revanche, l'elasticité des microfibrilles, qui, selon la théorie, ne fixent pas l'eau, ne varie pas au cours du séchage. Le rapport entre le module d'élasti- cité des microfibrilles et le module de rigidité de la matrice peut donc s'inverser au cours du séchage, entraînant un changement de signe de la déformation.

\section{CONCLUSIONS}

II est admis depuis longtemps que la densité du bois explique en partie la variabilité des retraits tranverses; les liaisons signalées par les expérimentateurs, qui comparent en général des arbres, des familles, voire des espèces entre elles sont rarement étroites. Nous montrons ici qu'au niveau de variabilité le plus bas, à l'intérieur de l'arbre, et en l'absence de bois de compression, la densité locale est fortement corrélée au retrait tangentiel (dont elle explique plus de $80 \%$ de la variabilité) et, dans une moindre mesure, au retrait longitudinal.

Dans une pièce hétérogène (bois massif, placage, panneau contreplaqué), la variabilité de densité entraîne la coexistence de zones denses à fort retrait tangentiel et de zones tendres à fort retrait longitudinal. Les déformations dues aux variations hygrometriques risquent d'être importantes dans le sens tangentiel en raison du fort module d'élasticité (densité forte) des zones à retrait élevé. Pour les emplois par déroulage, un taux minimal de fissuration du placage dans le bois dense serait souhaitable, de façon a diminuer le module d'élasticité tangentiel et, par là même, les risques d'ondulation. C'est d'ailleurs à une conclusion identique que parviennent, de façon empirique, Collet et al (1971): les placages de douglas déroulés avec un taux de compression trop élevé sont trop «rigides" et présentent, selon les auteurs, de fortes ondulations au séchage d'où 
il s'ensuit des problèmes de collage importants.

Dans le sens longitudinal, au contraire, les parties les plus denses se rétractent peu au cours du séchage et sont susceptibles de s'opposer à la déformation de l'ensemble, minimisant ainsi les risques d'ondulation dans cette direction. La présence de bois de compression peut alors avoir des conséquences catastrophiques puisque celui-ci présente simultanément un retrait longitudinal élevé et un fort module d'élasticité.

D'un point de vue expérimental, la $\mathrm{m}$ de mesure numérique des retraits longitudinal et tangentiel présentée ici paraît satisfaisante. Nous testerons dans une prochaine étude sa validité dans le cas d'un bois plus complexe de feuillu, en $y$ incorporant des informations sur les variations intracerne du retrait radial et en établissant les relations entre les retraits mesurés sur placage et les déformations d'une pièce de bois massif.

\section{RÉFÉRENCES}

Aleon D (1985) Mesure du retrait et de deformations au séchage du douglas de Normandie en vue de son utilisation en menuiserie intérieure, étude CTBA H214

Aubert M (1987) Recherches de relations entre caractéristiques simples du bois de Chêne rouge (Quercus rubra $L$ ) mesurées par voie non destructive et deux de ses propriétés technologiques: stabilité dimensionnelle des planches et qualité des placages d'ébénisterie. Thèse Nancy I

Barber NF, Meylan BA (1964) The anisotropic shrinkage of wood. Holzforshung 18 (5) 146-156

Boyd JD (1974) Anisotropic shrinkage of wood: identification of the dominant determinants. Mokuzai Gakkaishi 20, 473-482

CTBA (1986) Le douglas. Cahier 128

Collet BM, Brackley A, Cumming JD (1971) Simplified, highly accurate method of producting high-quality veneer. For Ind 62-64
El Osta M, Wellwood RW (1972) Short term creep as related to microfibril angle. Wood Fiber 4, 26-33

FPL (1960) Longitudinal shrinkage of wood. For Prod Lab Rept $n^{\circ} 1093$ For Serv USDA

Fujisaki K, Shibuya M (1986) On the relationship between anatomical features and the wood quality in the Sugi cultivars (2). Bull Ehime Univ For 24

Giordano $G$ (1959) Le caratteristiche del Legno di alcune resinose esotiche coltivate in Italia. Primo contributo: Pseudotsuga douglasii, Pinus strobus, Pinus canadiensis, Larix leptolepsis. Contributi scientifico-pratici per una migliore conoscenza ed utilizzazione del legno 29 (3), $44 p$

Hann RA (1969) Longitudinal shrinkage in seven species of wood. For Prod Lab Res note 0203, For Serv, USDA

Harris JM, Meylan BA (1965) The influence of microfibril angle on longitudinal and tangential skrinkage in Pinus radiata. Holzforschung 19, 144-153

Kollmann FFP, Cote WA Jr (1968) Principles of wood science and technology. I: Solid wood Springer-Verlag, New York

Lutz JF (1974) Techniques for peeling, slicing and drying veneer. FPL Madison Report 228

Luxeuil C (1983) Déformations du copeau de déroulage au séchage et hétérogénéité du bois due aux cernes. DEA Sciences du Bois, ENGREF, Nancy $40 \mathrm{p}$

Marchal R (1983) Intérêt de la prise en compte de caractéristiques physiques et anatomiques simples du bois de chêne pour l'appréciation de la qualité des placages d'ébénisterie. DEA Sciences du bois, INPL, Nancy

Marchal R (1989) Valorisation par tranchagedéroulage des chênes méditerranéens. These de doctorat en Sciences du Bois, INPL Nancy

Mothe $F$ (1988) Aptitude au déroulage du bois de douglas. Conséquences de l'hétérogéneité sur la qualité des placages. These de doctorat en sciences du bois, INPL, Nancy

Park SJ (1984) Structure of “Opposite» Wood. VII: Structure of "Opposite" wood formed in wide portion of annual rings. Mokuzai Gakkaishi 32, 293-298 
Pentoney RE (1953) Mechanisms affecting tangential VS radial shrinkage. J For Prod Res Soc 3 27-32

Polge $H$ (1963) Contribution à l'étude de la qualité du bois des principales essences résineuses exotiques dans les reboisements français. Ann Ecole Nat Eaux For 20, 404-469

Polge $H$ (1964) Le bois juvénile des conifères Rev For Fr 6, 475-505

Polge H, Keller R, Thiercelin F (1973) Influence de l'élagage de branches vivantes sur la structure des accroissements annuels et sur quelques caractéristiques du bois de douglas et de grandis. Ann Sci For 30, 127-140

Quirk JT (1982) Shrinkage and related properties of Douglas-fir cell walls. Wood Fiber Sci 16 (1), 115-133
Siripatanadilok.S, Leney L (1985) Compression wood in western hemlock (Tsuga heterophylla (Raf) sarg). Wood Fiber Sci 2, 254-265

Takahashi A, Tanaka C, Shiota Y (1983) Compilation of data on the mechanical properties of foreign woods. IV: European woods Facult Agric, Shimane Univ. Matsue, Jpn

Thibaut B (1988) Le processus de coupe du bois par déroulage. Thèse d'etat en sciences, université des sciences et techniques du Languedoc, Montpellier, $354 \mathrm{p}$

Timell TE (1986) Compression wood in gymnosperms. I: Bibliography, historical background, determination, structure, chemistry, topochemistry, physical properties, origin, and formation of compression wood. Springer-Verlag, Berlin 